\title{
Single-pass waveguide amplifiers in Er-Yb doped zinc polyphosphate glass fabricated with femtosecond laser pulses
}

\author{
Luke B. Fletcher, ${ }^{1, *}$ Jon J. Witcher, ${ }^{1}$ Neil Troy, ${ }^{1}$ Richard K. Brow, ${ }^{2}$ and Denise M. Krol ${ }^{1}$ \\ ${ }^{1}$ Department of Applied Science, University of California Davis, Davis, California 95616, USA \\ ${ }^{2}$ Department of Materials Science \& Engineering, Missouri University of Science and Technology, Rolla, Missouri 65409, USA \\ ${ }^{*}$ Corresponding author: lbfletcher@lbl.gov
}

Received September 15, 2011; revised January 25, 2012; accepted January 26, 2012; posted January 27, 2012 (Doc. ID 154643); published March 22, 2012

\begin{abstract}
We have investigated the direct fabrication of subsurface waveguide amplifiers in Er-Yb zinc polyphosphate glass by utilizing the relationship between the initial glass composition and the resulting changes to the network structure after modification by fs laser pulses. Waveguides, exhibiting internal gain of $1 \mathrm{~dB} / \mathrm{cm}$ at $1.53 \mu \mathrm{m}$ when pumped with $500 \mathrm{~mW}$ at $976 \mathrm{~nm}$, were directly fabricated using a regenerative amplified Ti:sapphire $1 \mathrm{kHz}, 180 \mathrm{fs}$ laser system. Optical properties as well as insertion losses and internal gain are reported. () 2012 Optical Society of America OCIS codes: $160.5690,160.2750,160.4760,350.3390$.
\end{abstract}

Tightly focused near-infrared femtosecond (fs) laser pulses can be used to create a permanent, micrometersized index modification $[\underline{1}-\underline{4}]$ inside a number of rareearth (RE) doped glass systems that can be used for the fabrication of active optical waveguide devices [5-15]. To date, waveguide amplifiers fabricated using the fs-laser direct-write technique have been reported in six different multicomponent RE-doped glass systems: Yb-doped phosphate glass (Kigre QX) [5], Er-Yb codoped phosphate glass (Kigre QX) [6-10], Nd-doped silicate glass [11], Er-doped oxyfluoride silicate glass [12,13], and $\mathrm{Er}-\mathrm{Yb}$ codoped oxyfluoride silicate glass [14]. Of all these active glasses, phosphate glass systems offer the best RE solubility without RE clustering and are ideal for the fabrication of compact waveguide amplifiers that operate in the telecommunications window $[15,16]$.

In many of these glasses, particularly phosphate glasses, the type of refractive index change that can be induced using the direct-write technique is determined by a combination of the laser processing conditions and the initial glass composition [8,10,17-21]. While commercially purchased QX phosphate glass has been engineered to function as a short-gain, solid-state laser medium that operates in the $C$ band, it is not necessarily the optimal material for fs-laser waveguide writing. We have recently reported [21] that fs-laser modification in an undoped zinc polyphosphate glass with an $O / P$ ratio of 3.25 (molar composition $60 \mathrm{ZnO}-40 \mathrm{P}_{2} \mathrm{O}_{5}$ ) always results in a change to the refractive index that is positive and ideal for the fabrication of optical waveguides over a wide range of fs-laser writing conditions. Using this result, it is possible to design an Er-Yb doped zinc polyphosphate glass with the same $O / P$ ratio in order to fabricate subsurface single-pass waveguide amplifiers. Optimization of the material composition results in an enhanced degree of flexibility with regards to waveguide processing conditions inside Er-Yb doped phosphate glass systems. In this letter we demonstrate the fabrication of a single-pass $1534 \mathrm{~nm}$ signal waveguide amplifier with fslaser inscription inside an Er-Yb doped phosphate glass $\left[0.65 \mathrm{Er}_{2} \mathrm{O}_{3}-1.30 \mathrm{Yb}_{2} \mathrm{O}_{3}-56.05 \mathrm{ZnO}-42.00 \mathrm{P}_{2} \mathrm{O}_{5}\right.$ (mol\%)] using a $1 \mathrm{kHz}$ repetition rate system. The Er-Yb doped zinc polyphosphate glass used in this study may be a more suitable material for directly fabricating waveguides under single-scan writing conditions over an expanded modification range. Such a unique property makes this glass an attractive substrate to use for fs-laser micromachining of active three-dimensional (3-D) photonic devices.

An Er-Yb codoped zinc polyphosphate glass with molar composition of $0.65 \mathrm{Er}_{2} \mathrm{O}_{3}-1.30 \mathrm{Yb}_{2} \mathrm{O}_{3}-56.05 \mathrm{ZnO}$ $42.00 \mathrm{P}_{2} \mathrm{O}_{5}$ (from here on referred to as $\mathrm{Er}-\mathrm{Yb} \mathrm{ZnP}$ ) was prepared using reagent-grade $\mathrm{ZnO}, \mathrm{Er}_{2} \mathrm{O}_{3}, \mathrm{Yb}_{2} \mathrm{O}_{3}$, and $\mathrm{NH}_{4} \mathrm{H}_{2} \mathrm{PO}_{4}$. A detailed melting procedure is described elsewhere [22]. Annealed samples, approximately $15 \mathrm{~mm} \times 5 \mathrm{~mm} \times 5 \mathrm{~mm}$ in size, were polished using $\mathrm{SiC}$ paper and diamond pastes to a finish of 0.25 microns.

Optical waveguides $1.5 \mathrm{~cm}$ in length were fabricated using linearly polarized light from a $800 \mathrm{~nm}, 1 \mathrm{kHz}, 180 \mathrm{fs}$ regeneratively amplified Ti:sapphire laser that was focused $200 \mathrm{~mm}$ below the surface of the glass sample. The slit-beam shaping method was used to minimize the strong focusing asymmetry experienced when using subsurface transverse writing techniques [23]. Fs laser pulse fluence, input beam dimensions, and sample translation speed were varied. For each set of processing conditions three identical waveguides were written, and excellent reproducibility was found. The best results, which are reported here, were obtained with the following writing parameters: sample translation speed of $5 \mu \mathrm{m} / \mathrm{s}$, pulse fluence of $10 \mathrm{~J} / \mathrm{cm}^{2}$, writing with a $300 \mu \mathrm{m}$-wide slit placed before a 0.55 numeric aperture (NA) focusing objective. The waveguides had a typical physical diameter of $11 \mu \mathrm{m}$, with a refractive index change of approximately $3 \times 10^{-3}$.

Figure 1 shows the setup that was used to measure single-pass gain in the waveguides. The waveguide amplifiers were bidirectionally pumped using a $976 \mathrm{~nm}$ singlemode diode laser capable of delivering up to $500 \mathrm{~mW}$ of output power to the glass sample.

The pump beam was split using a 50/50 cube beam splitter and directed into both ends of the waveguide using a series of mirrors, two identical beam splitters, and two identical 0.15 NA microscope objectives. The 


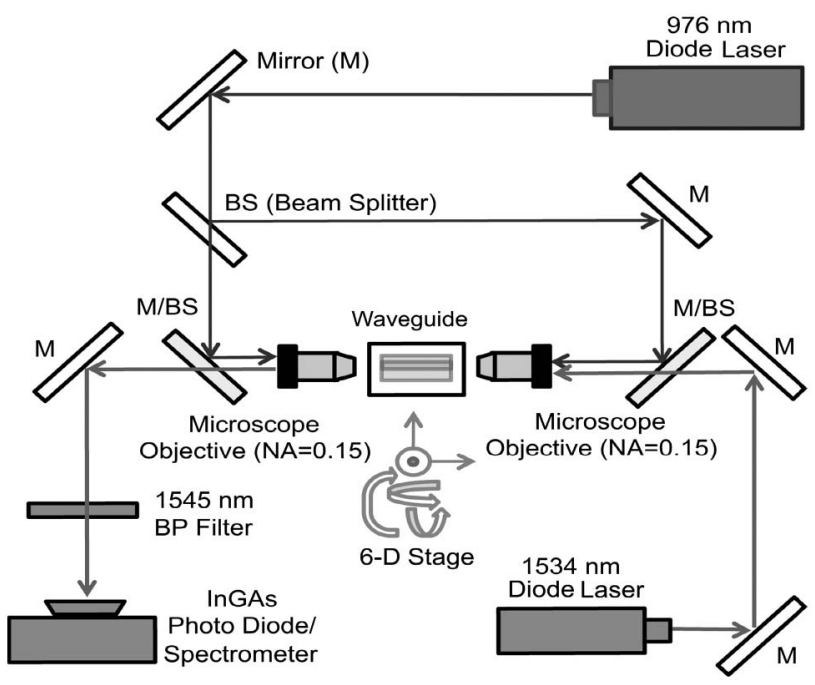

Fig. 1. Diagram of free-space waveguide amplifier setup.

backside-polished broadband dichroic mirror/beam splitters (Thorlabs BB1-EO3P) reflect $99.5 \%$ of $976 \mathrm{~nm}$ light at $45 \mathrm{deg}$ and transmit close to $85 \%$ of $1534 \mathrm{~nm}$ light at $45 \mathrm{deg}$. The $1534 \mathrm{~nm}$ signal was generated with a $1534 \mathrm{~nm}$ diode laser and overlapped with the $976 \mathrm{~nm}$ pump beam before the input microscope objective.

The spectrum and the power of the signal output were collected and measured from the output facet of the waveguide amplifier using an optical spectrum analyzer and an InGAs photodiode power meter, respectively. The two spectra in Fig. 2 show the Er-Yb absorption and emission spectrum measured in the bulk of the glass. It should be noted that the emission spectrum generated from $976 \mathrm{~nm}$ optical pumping of the waveguide is the same as that of the bulk. The optical emission and absorption properties are well suited for the fabrication of singlepass amplifiers that operate at $1534 \mathrm{~nm}[15,16]$.

The measured cross-sections and active ion densities, shown in Table 1, are very similar to those found in commercial Er-Yb doped Kigre QX phosphate glass $[\underline{9}, \underline{10}, \underline{18}]$. This allows for an appropriate comparison of the lasermaterial interactions and gain properties of the $\mathrm{Er}-\mathrm{Yb}$ codoped Kigre glass, which is widely used for fs-laser waveguide fabrication, to the $\mathrm{Er}-\mathrm{Yb} \mathrm{ZnP}$ glass that is used in this study.

Figure 3 shows the measured relative signal gain as a function of pump power at $976 \mathrm{~nm}$. The data show that an internal gain (at $1534 \mathrm{~nm}$ ) of approximately $1 \mathrm{~dB} / \mathrm{cm}$ was obtained with $500 \mathrm{~mW}$ pump power. This result is comparable to what has been previously reported in the literature for waveguide amplifiers fabricated in $\mathrm{Er}-\mathrm{Yb}$ doped QX phosphate glass (see Table $\underline{2}$ ) $[\underline{9}, \underline{10}, \underline{18}]$.

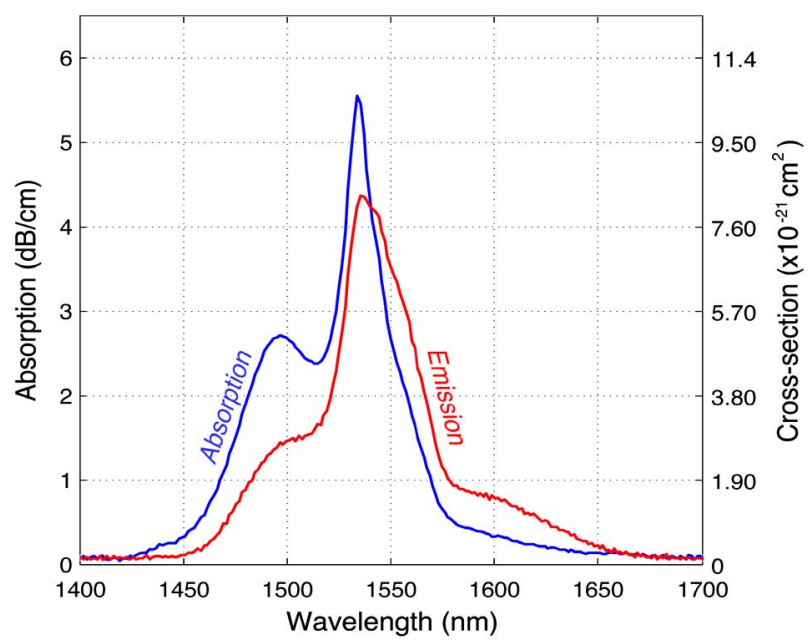

Fig. 2. (Color online) Absorption and emission spectrum of Er-Yb ZnP glass. Blue line, absorption spectrum (no $976 \mathrm{~nm}$ excitation, no $1534 \mathrm{~nm}$ signal); red line, emission spectrum under $976 \mathrm{~nm}$ excitation (no $1534 \mathrm{~nm}$ signal).

It is worth noting that, with a total waveguide length of $1.5 \mathrm{~cm}$, no net gain was observed for the amplifier setup used in this experiment. The measured internal gain was not sufficient to overcome the total insertion losses of the system. Table 2 shows a breakdown of the different contributions to the overall amplifier loss, comparing our $\mathrm{ZnP}$ waveguide amplifier to the Kigre QX amplifier. The data clearly indicate that the largest difference between these two amplifiers is in the coupling loss, with a coupling loss of $4.15 \mathrm{~dB}$ in the $\mathrm{ZnP}$ system versus $0.2 \mathrm{~dB}$ in the Kigre QX system. This huge difference is due to the fact that our amplifier uses free-space coupling, which suffers from poor matching between NA and spot size, as opposed to fiber coupling in the Kigre QX system. Future experiments will focus on decreasing these coupling losses by optimization of the pump power configuration and signal coupling via the use of optical fibers. Other efforts to improve our device performance involve the use of samples of greater length and higher optical quality. This can be achieved by improved melting procedures to obatin larger samples with reduced propagation losses due to absorption from impurities and scattering from density fluctuations. The ideal physical length of the waveguide amplifier, given the optical properties of this substrate, is approximately $5 \mathrm{~cm}[9,24]$.

In terms of the net induced $\Delta n$ and the internal gain (Table 2) our ZnP glass waveguides currently show similar specifications compared to waveguides fabricated in Er-Yb QX phosphate glass using $1 \mathrm{kHz}, 800 \mathrm{~nm}, 120 \mathrm{fs}$ and $150 \mathrm{fs}$ laser systems $[9,10,18,24]$. The internal gain of our glass sample can be enhanced by reducing propagation

Table 1. Glass Properties ${ }^{a}$

\begin{tabular}{ccccc}
\hline & $\mathrm{N}\left[\mathrm{Er}^{3+}\right]$ & $\mathrm{N}\left[\mathrm{Yb}^{3+}\right]$ & $\sigma_{E}$ & $\sigma_{A}$ \\
\cline { 2 - 5 } Glass Sample & $\left(\mathrm{ions} / \mathrm{cm}^{3}\right) \times 10^{20}$ & $\times 10^{-20} \mathrm{~cm}^{-2}$ & $\rho\left(\mathrm{g} / \mathrm{cm}^{3}\right)$ \\
\hline Er-Yb ZnP1.9\% wt. $\mathrm{Er}^{3+} 4.0 \%{\text { wt. } \mathrm{Yb}^{3+}}^{3+}$ & 2.0 & 4.0 & 8.3 & 1.7 \\
Kigre QX $\%$ wt. $\mathrm{Er}^{3+} 4 \%$ wt. $\mathrm{Yb}^{3+}$ & 1.8 & 3.6 & 8.0 & 1.7 \\
\hline
\end{tabular}

${ }^{a}$ Data from Kigre Inc. 
Table 2. Modification and Amplifier Comparison ${ }^{a}$

\begin{tabular}{cccccc}
\hline Glass & $\Delta n \times 10^{-3}$ & $\mathrm{CL}(\mathrm{dB})$ & $\mathrm{PL}(\mathrm{dB} / \mathrm{cm})$ & Modif. Range $\times 10^{13}\left(\mathrm{~W} / \mathrm{cm}^{2}\right)$ & Internal Gain $500 \mathrm{~mW}$ pump \\
\hline Kigre QX & 3.5 & $0.2^{\mathrm{a}}$ & $0.3 @ 1600 \mathrm{~nm}$ & $1.67-4.17$ & $1 \mathrm{~dB} / \mathrm{cm}^{\mathrm{a}}$ \\
Er-Yb ZnP & 3 & 4.15 & $0.54 @ 633 \mathrm{~nm}$ & $1.11-8.33$ & $1 \mathrm{~dB} / \mathrm{cm}$ \\
\hline
\end{tabular}

${ }^{a}$ Fiber-coupled amplifier setup. CL, coupling; PL, propagation loss.

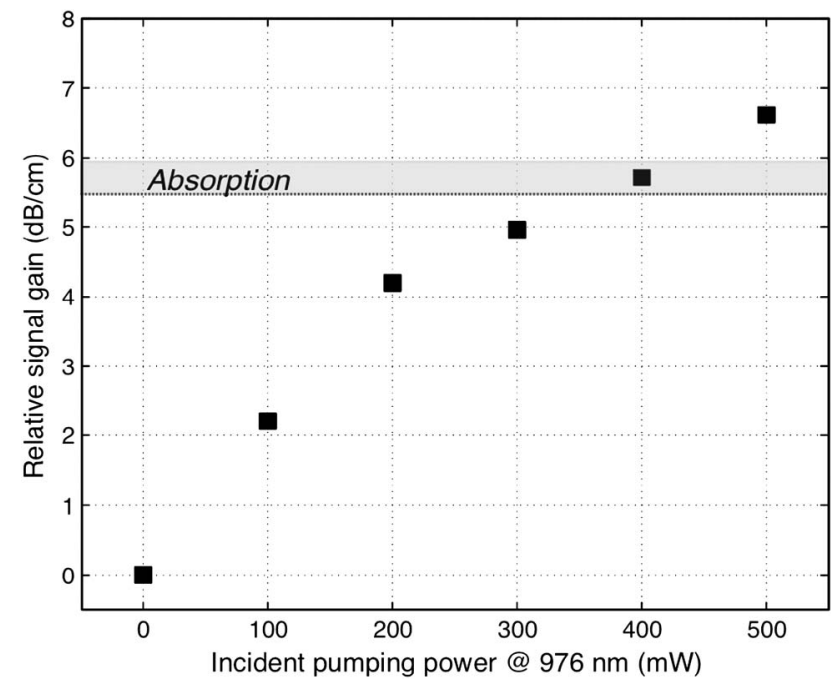

Fig. 3. Signal enhancement at $1534 \mathrm{~nm}$ versus total bidirectional $976 \mathrm{~nm}$ pump power; grey line: total absorption of $\mathrm{Er}-\mathrm{Yb} \mathrm{ZnP}$ glass.

losses as described above. In addition, the $\mathrm{ZnP}$ glass benefits from a wider range of writing conditions that could be used to fabricate waveguides as well as the ability to manufacture comparable devices under single-scan writing conditions. This allows for less stringent requirements in terms of the mechanical stability of the writing stage, better control of the change in the refractive index, and thus more flexibility in the fs-laser processing of active photonics devices.

In conclusion, we have fabricated low-loss, singlemode waveguides inside Er-Yb doped zinc polyphosphate glass $\left(0.65 \mathrm{Er}_{2} \mathrm{O}_{3}-1.30 \mathrm{Yb}_{2} \mathrm{O}_{3}-56.05 \mathrm{ZnO}\right.$ $-42.00 \mathrm{P}_{2} \mathrm{O}_{5}$ ) using a $1 \mathrm{kHz}$ fs laser. By optically pumping the waveguides with a maximum $500 \mathrm{~mW}$ of $976 \mathrm{~nm}$ pump power, a total $1534 \mathrm{~nm}$ signal enhancement of approximately $10 \mathrm{~dB}$ was measured over a $1.5 \mathrm{~cm}$ length, resulting in an internal gain of close to $1 \mathrm{~dB} / \mathrm{cm}$. Results from this study demonstrate the ability to fabricate a freespace, single-pass $1534 \mathrm{~nm}$ signal waveguide amplifier with fs-laser waveguide writing. Moreover, the $\mathrm{Er}-\mathrm{Yb}$ doped zinc polyphosphate glass was a suitable active material that could be used to fabricate waveguides and amplifiers using a $1 \mathrm{kHz}$ repetition rate fs-laser system with comparable device specifications to other waveguide amplifying devices fabricated inside commercially purchased $\mathrm{Er}-\mathrm{Yb}$ doped phosphate glass.

This work was supported by the National Science Foundation (NSF) grant DMR 0801786 and grant CMMI 0825572 .

\section{References}

1. K. M. Davis, K. Miura, N. Sugimoto, and K. Hirao, Opt. Lett. 21, 1729 (1996).

2. E. N. Glezer and E. Mazur, Appl. Phys. Lett 71, 882 (1997).

3. R. R. Gattass and E. Mazur, Nat. Photon. 2, 219 (2008).

4. K. Itoh, W. Watanabe, S. Nolte, and C. Schaffer, MRS Bull. 31, 620 (2006).

5. G. D. Marshall, P. Dekker, M. Ams, J. A. Piper, and M. J. Withford, Opt. Lett. 33, 956 (2008).

6. R. Osellame, S. Taccheo, G. Cerullo, M. Marangoni, D. Polli, R. Ramponi, P. Laporta, and S. De Silvestri, Electron. Lett. 38, 964 (2002).

7. S. Taccheo, G. Della Valle, R. Osellame, G. Cerullo, N. Chiodo, P. Laporta, O. Svelto, A. Killi, U. Morgner, M. Lederer, and D. Kopf, Opt. Lett. 29, 2626 (2004).

8. R. Osellame, N. Chiodo, G. Della Valle, S. Taccheo, R. Ramponi, G. Cerullo, A. Killi, U. Morgner, M. Lederer, and D. Kopf, IEEE J. Sel. Top. Quantum Electron. 12, 277 (2006).

9. R. Osellame, G. D. Valle, N. Chiodo, S. Taccheo, P. Laporta, O. Svelto, and G. Cerillo, Appl. Phys. A 93, 17 (2008).

10. G. D. Valle, R. Osellame, and P. Laporta, J. Opt. 11, 013001 (2009).

11. Y. Sikorski, A. A. Said, P. Bado, R. Maynard, C. Florea, and K. A. Winick, Electron. Lett. 36, 226 (2000).

12. R. R. Thomson, S. Campbell, I. J. Blewett, A. K. Kar, D. T. Reid, S. Shen, and A. Jha, Appl. Phys. Lett. 87, 121102 (2005).

13. R. R. Thomson, H. T. Bookey, N. Psaila, S. Campbell, D. T. Reid, S. X. Shen, A. Jha, and A. K. Kar, IEEE Photon. Technol. Lett. 18, 1515 (2006).

14. N. D. Psaila, R. R. Thomson, H. T. Bookey, A. K. Kar, N. Chiodo, R. Osellame, G. Cerullo, A. Jha, and S. Shen, Appl. Phys. Lett. 90, 131102 (2007).

15. P. Laporta, S. Taccheo, S. Longhi, O. Svelto, and C. Svelto, Opt. Mater. 11, 269 (1999).

16. M. J. Weber, J. Non-Cryst. Solids 123, 208 (1990).

17. L. B. Fletcher, J. J. Witcher, W. B. Reichman, A. Arai, J. Bovatsek, and D. M. Krol, J. Appl. Phys. 106, 083107 (2009).

18. M. Ams, G. D. Marshall, P. Dekker, M. Dubov, V. K. Mezentsev, I. Bennion, and M. J. Withford, IEEE J. Sel. Top. Quantum Electron. 14, 1370 (2008).

19. D. Esser, D. Mahlmann, D. Wortmann, and J. Gottmann, Appl. Phys. B 96, 453 (2009).

20. A. Ferrer, A. R. de la Cruz, D. Puerto, W. Gawelda, J. A. Vallés, M. A. Rebolledo, V. Berdejo, J. Siegel, and J. Solis, J. Opt. Soc. Am. B 27, 1688 (2010).

21. L. B. Fletcher, J. J. Witcher, N. Troy, S. T. Reis, R. K. Brow, and D. M. Krol, Opt. Express 19, 7929 (2011).

22. L. B. Fletcher, J. J. Witcher, N. Troy, S. T. Reis, R. K. Brow, R. M. Vazquez, R. Osellame, and D. M. Krol, Opt. Mater. Express 1, 845 (2011).

23. M. Ams, G. D. Marshall, D. J. Spence, and M. J. Withford, Opt. Express 13, 5676 (2005).

24. J. A. Vallés, M. Rebolledo, V. Berdejo, A. Ferrer, A. Ruiz de la Cruz, and J. Solís, Opt. Mater. 33, 231 (2010). 